\title{
COMBINAÇÃO DE ABORDAGENS GLSP E ATSP PARA O PROBLEMA DE DIMENSIONAMENTO E SEQUENCIAMENTO DE LOTES DE PRODUÇÃO DE SUPLEMENTOS PARA NUTRIÇÃO ANIMAL
}

\author{
Eli Angela Vitor Toso \\ Reinaldo Morabito* \\ Departamento de Engenharia de Produção \\ Universidade Federal de São Carlos (UFSCar) \\ São Carlos - SP \\ eli@ufscar.br; morabito@ufscar.br
}

\author{
Alistair Clark \\ Fac. of Computing, Engineering and Mathematical Sciences \\ University of the West of England (UWE) \\ Frenchay Campus - Bristol, England \\ alistair.clark@uwe.ac.uk \\ * Corresponding author / autor para quem as correspondências devem ser encaminhadas \\ Recebido em 04/2007; aceito em 06/2008 \\ Received April 2007; accepted June 2008
}

\section{Resumo}

Neste artigo estudamos a combinação de abordagens GLSP (General Lot Sizing and Scheduling Problem) e ATSP (Asymmetric Travelling Salesman Problem) para o problema de dimensionamento e sequenciamento de lotes na indústria de nutrição animal. Este problema consiste em determinar o tamanho de cada lote de produção para cada produto, assim como a sequência de produção destes lotes, de forma a satisfazer a demanda sem atrasos e minimizar os custos de produção e estoques. Uma dificuldade para a programação da produção nesta indústria é integrar estas decisões, pois os tempos de preparação da linha de produção são dependentes da sequência produtiva e não obedecem a desigualdade triangular. A abordagem proposta é comparada com abordagens relax-and-fix para o modelo GLSP (General Lot-sizing and Scheduling Problem) estudadas em trabalhos anteriores, utilizando dados reais de um estudo de caso de uma fábrica de nutrição animal localizada no interior de São Paulo.

Palavras-chave: dimensionamento de lotes; sequenciamento da produção; indústria de nutrição animal; ATSP; GLSP.

\begin{abstract}
In this paper we study the combination of GLSP (General Lot Sizing and Scheduling Problem) and ATSP (Asymmetric Travelling Salesman Problem) approaches with sub-tour elimination and patching to a lot sizing and sequencing problem in the animal nutrition industry. This problem consists of deciding the lots size for each product as well the production sequence of the lots, while meeting demand without backlogs and minimizing production and inventory costs. The coordination of these decisions is a challenge for production scheduling in this industry as the setup times are sequence dependent. The ATSP approaches are compared with relax-and-fix approaches applied to the GLSP (General Lot-sizing and Scheduling Problem) formulated in previous research, using real data from an animal nutrition plant in Sao Paulo state.
\end{abstract}

Keywords: lot sizing; scheduling; production sequencing; animal nutrition industry; ATSP; GLSP. 


\section{Introdução}

A indústria de produtos para nutrição animal é uma das que mais cresce em todo mundo, impulsionada principalmente pelas preocupações com a segurança alimentar. De acordo com IFIF (2008) (International Feed Industry Federation - Federação Internacional das Indústrias de Alimentação Animal), a produção mundial de rações vem crescendo a níveis recordes, atualmente são produzidas cerca de 614 milhões de toneladas por ano. A produção brasileira de alimentos balanceados para nutrição animal é a terceira maior do mundo, com perspectivas de crescimento (SINDIRAÇÕES, 2008). De acordo com levantamentos setoriais (FINEP, 2004), dentre os principais elementos competitivos nesta indústria estão a eficiência produtiva e os preços. Desta forma, as empresas do setor encontram-se em constante busca por novas tecnologias, aperfeiçoamento dos sistemas de produção e otimização da utilização dos recursos produtivos.

Algumas peculiaridades do processo produtivo desta indústria desafiam a tomada de decisões do planejamento, programação e controle da produção, principalmente as decisões relacionadas ao dimensionamento e sequenciamento de lotes de produção. $\mathrm{O}$ dimensionamento de lotes nesta indústria consiste em determinar quanto produzir de cada produto em cada período, ajustando a capacidade produtiva num ambiente de demanda dinâmica. $\mathrm{O}$ sequenciamento de lotes consiste em determinar em que ordem produzir estes lotes de forma a minimizar o total dos tempos de preparação, que são dependentes da sequência produtiva. Um sequenciamento ruim pode reduzir significativamente a capacidade da linha de produção. Durante o processamento, alguns produtos deixam resíduos que podem contaminar a linha de produção. Em geral, estes produtos têm maior valor agregado e baixa demanda. $\mathrm{O}$ ideal seria agregar os lotes de produção destes produtos para economizar tempos de preparação; entretanto, as incertezas em relação à demanda e custos de estocagem são fatores de risco nesta estratégia.

Conforme discutido em um trabalho anterior (Toso \& Morabito, 2005), na prática, as decisões de dimensionamento e sequenciamento de lotes nesta indústria são frequentemente tomadas de maneira separada. Isto pode comprometer tanto a qualidade das decisões em relação aos custos operacionais e atendimento dos prazos de entrega, quanto o tempo de resposta da empresa às constantes variações de demanda, que acarretam na necessidade de reavaliação periódica de planejamento. $\mathrm{O}$ uso de modelos quantitativos para o problema de dimensionamento e sequenciamento de lotes na indústria de nutrição animal é bastante promissor, uma vez que permite integrar as decisões envolvidas e melhorar a qualidade das soluções obtidas. Entretanto, a representação matemática do problema envolve o uso de muitas variáveis de decisão discretas, resultando em modelos de programação linear inteira mista em geral de difícil resolução exata em tempo computacional aceitável.

O objetivo do presente trabalho é apresentar abordagens de solução viáveis para o problema de dimensionamento e sequenciamento de lotes de produção da indústria de nutrição animal, combinando as formulações do modelo Genérico de Dimensionamento e Sequenciamento de Lotes (GLSP) com formulações do caixeiro viajante assimétrico (ATSP - Asymmetric Travelling Salesman Problem) para modelar as decisões de sequenciamento. Por simplicidade, neste artigo denominamos de abordagem ATSP. Inicialmente, considera-se no modelo cada período do horizonte de planejamento de maneira independente (admitindo-se uma limpeza da linha no final de cada período) e, depois, considera-se todos os períodos de maneira dependente, incluindo variáveis para guardar a sequência entre os períodos (carryover). Para resolução destes modelos são utilizados dois métodos: eliminação de subrotas; e, eliminação e combinação de sub-rotas (patching). 
O presente artigo está organizado da seguinte maneira: na próxima seção o problema de dimensionamento e sequenciamento de lotes na indústria de suplementos para nutrição animal é resumidamente descrito. Na seção 3 revisam-se brevemente modelos e métodos para resolver problemas de dimensionamento e sequenciamento de lotes com setup dependente da sequência. Na seção 4 o problema aplicado na indústria de nutrição animal é modelado, primeiro considerando períodos com sequências independentes (modelo ATSPperíodos) e, depois, como um ATSP com períodos com sequências dependentes (modelo ATSP-carryover). Na seção 5 são apresentadas abordagens de solução para os modelos ATSP, baseadas em eliminação de sub-rotas e combinação de sub-rotas (patching). Na seção 6 são apresentados e analisados os resultados computacionais obtidos com a aplicação das abordagens em exemplares reais de um estudo de caso de uma fábrica de nutrição animal localizada no interior de São Paulo. Finalmente na seção 7 são discutidas as conclusões deste estudo e as perspectivas para pesquisa futura.

\section{A Indústria de Nutrição Animal}

A indústria de nutrição animal engloba fábricas de rações balanceadas, fábricas de suplementos (núcleos, premixes e minerais) e fábricas de ingredientes ou matérias primas específicas para alimentação animal. De acordo com Brito \& Stringhini (2002), os processos básicos para fabricação de ração compreendem: recepção, transformação, preparação e finalização. A recepção envolve o recebimento e armazenagem das matérias primas. A transformação consiste em operações de pré-limpeza, secagem e moagem dos grãos (quando necessário). A preparação compreende as operações de dosagem, mistura e peletização ou extrusão. A finalização consiste nas operações de ensaque e expedição. As operações podem ser combinadas, obtendo-se diferentes processos de produção. Em geral, os possíveis fluxos de produção dependem da apresentação do produto, ou seja, seu formato específico. As rações prontas para consumo podem ser fareladas, trituradas, extrusadas ou peletizadas. Já os premixes, núcleos e suplementos geralmente são produtos farelados.

Este trabalho está baseado no estudo da unidade produtora de suplementos de uma empresa do setor, que produz suplementos vitamínicos: sais minerais, núcleos e premixes para bovinos, equinos, suínos e aves. Embora o trabalho esteja baseado no estudo de um único caso, pode ser estendido para outras unidades de produção na mesma empresa, bem como para outras empresas fabricantes de ração, e ainda para processos produtivos similares como, por exemplo, em algumas indústrias alimentícias (cereais matinais, massas, produtos de panificação, etc).

$\mathrm{O}$ processo de produção é intermitente e ocorre em bateladas. Cada batelada de produção corresponde ao lote mínimo produzido em cada operação. A quantidade produzida em cada batelada é limitada pelo tamanho do misturador e varia de um produto para outro, dependendo da densidade de cada produto. Embora o processo produtivo tenha várias etapas, para os propósitos de modelagem, pode ser considerado mono estágio. A capacidade produtiva é determinada como o tempo disponível para produção.

A demanda tem características sazonais, ou seja, ao longo do ano existem variações em relação aos tipos de produtos em cada período (mix) e variações em relação às quantidades produzidas. Para ajustar a capacidade produtiva a estas oscilações da demanda, a empresa adota a estratégia de utilizar horas extras nos períodos de pico, ao invés de antecipar a produção e carregar estoques de um período para outro. Esta decisão é fortemente justificada

Pesquisa Operacional, v.28, n.3, p.423-450, Setembro a Dezembro de 2008 
pela perecibilidade dos produtos. Vale ressaltar que esta decisão precede as decisões de dimensionamento e sequenciamento de lotes, as quais são tomadas num contexto de curto prazo. Desta forma, a perecibilidade dos produtos não é considerada neste horizonte de planejamento mais curto, em geral de um mês, desdobrado semanalmente.

A cada semana as previsões de demanda mensais vão sendo reavaliadas, considerando-se novos pedidos, possíveis cancelamentos de pedidos em carteira, a capacidade disponível e a necessidade de utilização de horas extras. Com isso são determinados os tamanhos dos lotes em cada semana. Uma vez determinados estes tamanhos, são emitidas ordens de fabricação e o sequenciamento dos lotes é realizado por um supervisor de produção no chão de fábrica. Como algumas formulações misturam diversos tipos de medicamentos e minerais, é necessário sequenciar os lotes de forma a evitar que produtos com agentes contaminantes deixem resíduos na linha de produção, comprometendo a qualidade do próximo lote. Para evitar isto, há duas alternativas: procurar uma sequência em que nenhum produto contamine os demais ou, quando isso não é possível, fazer uma limpeza nos equipamentos (setup), o que consome um longo tempo de produção.

$\mathrm{Na}$ prática, o dimensionamento de lotes é feito considerando a capacidade produtiva em termos das horas disponíveis para produção no mês e de uma taxa média de produção por hora. Não é considerado o fato de que os tempos de preparação são dependentes da sequência produtiva. Ou seja, diferentes programas resultam em diferentes sequências, sendo que algumas podem demandar maior consumo de capacidade devido à necessidade de mais preparações, podendo inviabilizar alguns programas de produção. Portanto, a empresa frequentemente tem dificuldades em coordenar de forma eficaz o dimensionamento de lotes com o sequenciamento da produção, pois, uma vez definidos os tamanhos dos lotes, pode não ser possível encontrar uma sequência de produção que seja viável do ponto de vista da capacidade disponível.

\section{Revisão Bibliográfica}

Não foram encontrados na literatura trabalhos sobre planejamento e programação da produção especificamente na indústria de nutrição animal, exceto trabalhos sobre o problema da mistura, onde o problema da ração é um exemplo clássico. A maioria dos trabalhos sobre esta indústria, tanto no Brasil quanto no mundo, são artigos focados nos aspectos técnicos de produção animal: genética, nutrição, etc. A seguir revisamos modelos de dimensionamento de lotes e sequenciamento da produção, bem como modelos integrados, que podem ser considerados para representar processos decisórios desta indústria, e também algumas reformulações destes modelos baseadas no ATSP.

\subsection{Modelos de Dimensionamento de Lotes e Programação da Produção}

Diversos modelos e métodos de solução para o problema de dimensionamento de lotes (lot sizing) e para programação da produção (scheduling) podem ser encontrados em Johnson \& Montgomery (1974), Graham et al. (1979), Hax \& Candea (1984), Maes \& Wassenhove (1988), Trigeiro et al. (1989), Winston (1991), Askin \& Standridge (1993), Williams (1993), Graves et al. (1993), Lawler et al. (1993), Gershwin (1994), Pinedo (1995), Nahmias (1995), Blazewicz et al. (1996), Drexl \& Kimms (1997), Allahverdi et al. (1999), Potts \& Kovalyov (2000), Karimi et al. (2003), Pochet \& Wolsey (2006). Em geral, os modelos apresentados se 
diferem quanto à limitação de capacidade, quanto ao comportamento da demanda, aos estágios do processo de fabricação, ao número de itens, aos objetivos específicos de cada problema, a estrutura de custos, ao horizonte de planejamento, etc.

Por exemplo, em Johnson \& Montgomery (1974), Hax \& Candea (1984), Maes \& Wassenhove (1988) e Trigeiro et al. (1989) são tratados apenas problemas de dimensionamento de lotes, enquanto que em Pinedo (1995), Blazewicz et al. (1996), Lawler et al. (1993), Allahverdi et al. (1999) e Potts \& Kovalyov (2000) são encontrados modelos de programação da produção. Conforme Drexl \& Kimms (1997) e Karimi et al. (2003), a tendência é combinar o problema de dimensionamento de lotes às decisões de programação da produção (lot-sizing and scheduling problems, ou simplesmente lot-scheduling). Estes autores apresentam uma revisão destes problemas integrados.

Drexl \& Kimms (1997) consideram os modelos capacitados, dinâmicos e determinísticos, apresentando as formulações matemáticas, e uma comparação entre vários modelos como: CLSP (Capacitated Lot Sizing Problem), DLSP (Discrete Lot-sizing and Scheduling Problem), CSLP (Continuous Setup Lot-sizing Problem), PLSP (Proporcional Lot-sizing and Scheduling Problem), GLSP (General Lot-sizing and Scheduling Problem), etc. O artigo de Karimi et al. (2003) focaliza o CLSP, citando os demais modelos como variantes e apresentando os métodos de solução estudados a partir da seguinte classificação: métodos exatos, métodos heurísticos (baseados no conhecimento do problema) e heurísticas baseadas em programação matemática.

Pochet \& Wolsey (2006) apresentam diferentes modelos para o planejamento e programação da produção, bem como métodos de otimização baseados em programação inteira mista $\mathrm{e}$ técnicas de reformulação para melhorar os modelos matemáticos. Diversos outros trabalhos abordando o problema integrado em diferentes variações, enfoques e métodos de solução são encontrados na literatura, como, por exemplo, Fleishmann (1994), Drexl \& Haase (1995), Haase (1996), Salomon et. al. (1997), Fleischmann \& Meyr (1997), França et al. (1997), Laguna (1999), Armentano et al. (1999), Haase \& Kimms (2000), Meyr (2000), Clark \& Clark (2000), Meyr (2002), Clark (2003), Araujo \& Arenales (2003), Rangel \& Ferreira (2003), Araujo et al. (2004), Luche \& Morabito (2005), Toledo \& Armentano (2006), Toledo et al. $(2007,2008)$ e Ferreira et al. (2008a, 2008b).

\subsection{Modelos e métodos baseados em ATSP}

Devido à complexidade computacional dos problemas de dimensionamento e sequenciamento de lotes, existem na literatura diversas propostas de métodos de solução. Dentre elas, destacamos algumas em que o modelo é reformulado com base no problema de caixeiro viajante. Este problema é amplamente estudado na literatura e pode ser definido da seguinte forma: dado um grafo orientado simples $\mathrm{G}=(V, A)$, onde $V$ é o conjunto de $n$ vértices e $A$ um conjunto de $m$ arcos ou arestas, tal que a cada arco $(i, j)$ está associado um custo $c_{i j}$, o problema consiste em determinar em $\mathrm{G}$ um ciclo Hamiltoniano de custo mínimo. Num grafo orientado, um ciclo Hamiltoniano é um ciclo com todos os arcos orientados na mesma direção e que passa por todos os vértices uma única vez. No caso assimétrico (ATSP), podemos ter $c_{i j} \neq c_{j i}, i, j \in V$ (por exemplo, Laporte, 1992).

Algumas revisões sobre modelos e métodos para o ATSP podem ser encontrados em Lawler et al. (1985), Laporte (1992), Junger et al. (1995), Carpaneto et al. (1995), Zhang (1997), Glover et al. (2001), Cirasella et al. (2001), Johnson et al. (2002), Buriol et al. (2003), 
Orman \& Williams (2004). As aplicações mais comuns do ATSP estão associadas a problemas de roteamento. No entanto, existem outros problemas que podem ser interpretados como ATSP, entre eles o sequenciamento de tarefas.

Fleishmann (1994) apresenta uma formulação para o DLSP incluindo custos de preparação dependentes da sequência; depois reformula o problema usando o ATSP com janelas de tempo, e propõe um procedimento heurístico para solução. Salomon et al. (1997) formulam um problema semelhante que inclui, além dos custos, os tempos de preparação. Os nós do grafo representam as demandas de cada produto para cada período, bem como a demanda por períodos ociosos, e as janelas de tempo garantem que não ocorram atrasos. O problema é resolvido por meio de um método ótimo baseado em programação dinâmica. Pochet \& Wolsey (2006) também sugerem a utilização da formulação ATSP para modelar problemas com vários itens, único período, com variáveis dependentes da sequência.

Clark \& Clark (2000) desenvolveram um modelo para o problema capacitado, multi-item, monoestágio, com tempos de preparação dependentes da sequência, considerando que os produtos podem ser produzidos em máquinas paralelas, e com permissão de pedidos atrasados (backorder). Em um trabalho posterior, Clark (2003) apresenta um método de resolução em que o problema é decomposto: as decisões de dimensionamento de lotes resultam em um modelo linear; enquanto que as decisões de sequenciamento são remodeladas usando a formulação ATSP. Diferentemente dos trabalhos de Fleischmann (1994) e Fleischmann \& Meyr (1997), que apresentam a formulação matemática para o DLSP e depois definem o problema como TSP, o trabalho de Clark (2000) reformula matematicamente o problema, usando equações clássicas do problema do caixeiro viajante para representar as decisões do sequenciamento de lotes.

Um método bem sucedido para resolver otimamente problemas ATSP de tamanho moderado consiste em: resolver rapidamente o problema sem restrições de sub-rotas (o que fornece um limitante inferior); identificar as sub-rotas resultantes nesta solução; e, então resolver o problema novamente proibindo explicitamente as sub-rotas identificadas (Lawler et al., 1985; Laporte \& Nobert, 1980; Padberg \& Rinaldi, 1991; Applegate et al., 2003). O método trabalha iterativamente desta maneira até obter uma solução que não contenha nenhuma subrota. Para acelerar a convergência do método, pode ser usado um procedimento heurístico, baseado na combinação das sub-rotas em uma única rota a cada iteração, fornecendo uma solução factível e um limitante superior (Karp, 1979; Karp \& Steele, 1985; Frieze \& Dyer, 1990; Pekny \& Miller, 1990; Frieze et al., 1995; Carpaneto et al., 1995; Zhang, 1993; Zhang, 1997; Glover et al., 2001; Gutin \& Zverovich, 2000; Cirasella et al., 2001).

\subsection{O método relax-and-fix aplicado ao modelo GLSP}

Wolsey (1998) discute um método heurístico para problemas de programação inteira mista (MIP) chamado relax-and-fix, no qual o problema é resolvido de forma iterativa. As variáveis são divididas em dois grupos, onde um representa as variáveis de decisão mais importantes, por exemplo, as que envolvem maiores investimentos ou as que representam as decisões mais imediatas. Desta forma, uma sequência de MIPs parcialmente relaxados é resolvida, cada um com um grupo reduzido de variáveis inteiras, cujo número é pequeno o bastante para obter rapidamente soluções ótimas MIP (Clark \& Clark, 2000). Conforme a série avança, cada grupo de variáveis inteiras é permanentemente fixado em seus valores da solução. 
Em Toso et al. (2007), este método é aplicado ao modelo GLSP para indústria de nutrição animal, onde as variáveis são divididas de duas formas:

Relax-and-fix variáveis. As variáveis inteiras e binárias são divididas em dois grupos: variáveis de dimensionamento de lotes e variáveis de preparação. Em um primeiro passo da heurística relax-and-fix, as variáveis de dimensionamento são relaxadas e o modelo GLSP é resolvido. Os valores obtidos para as variáveis de preparação são fixados, a condição de integralidade das variáveis de dimensionamento é retomada, e o modelo é resolvido novamente.

Relax-and-fix períodos. As variáveis inteiras e binárias são divididas por períodos. Em um primeiro passo somente as variáveis do primeiro período são mantidas inteiras ou binárias, as variáveis dos demais períodos são relaxadas, e o modelo GLSP é resolvido. Os valores obtidos para as variáveis do primeiro período são fixados, as condições de integralidade são retomadas para as variáveis do segundo período, e o modelo GLSP é novamente resolvido. Este procedimento é repetido até que todos os períodos tenham sido resolvidos - ele pode ser feito do primeiro para o último período (forward), ou do último para o primeiro (backward).

\section{Modelagem do problema}

Para reformular o modelo GLSP de nutrição animal (Toso \& Morabito, 2005; Toso et al., 2007) usando a formulação ATSP, mantemos as mesmas considerações dos trabalhos anteriores:

- A unidade de produção é uma batelada, independente do seu tamanho. As demandas por produto são agregadas e aproximadas pelos múltiplos dos tamanhos das bateladas;

- Os produtos são agregados em famílias, onde cada família só tem produtos pertencentes ao mesmo grupo de contaminação e com características comuns, como tempo de processamento e quantidade por batelada;

- Quando não existe risco de contaminação residual, o tempo de preparação entre um lote e outro é pequeno e perto de zero. Por simplicidade, os tempos de preparação pequenos são desprezados.

Neste modelo considera-se que cada família é produzida uma única vez em cada período. Esta é uma diferença significativa entre os modelos. Baseadas nas estratégias da empresa para os diferentes ciclos sazonais, são apresentadas a seguir duas abordagens: uma onde é resolvido um ATSP para cada período (sequências dos períodos admitidas independentes), e outra onde é resolvido um único ATSP para todo o horizonte de planejamento (sequências de todos os períodos dependentes).

\subsection{ATSP - Períodos com Sequências Independentes}

Nesta abordagem pressupõe-se que é feita uma limpeza ao final de cada período, por exemplo, no final de cada semana. Desta forma, uma nova sequência começa sem nenhuma relação com a anterior, do ponto de vista do sequenciamento. Assim, o dimensionamento e o sequenciamento são feitos de forma simultânea para todo horizonte de planejamento, mas as sequências em cada período são independentes entre si. A empresa estuda a possibilidade de adotar esta estratégia devido às pressões do mercado por produtos seguros.

Pesquisa Operacional, v.28, n.3, p.423-450, Setembro a Dezembro de 2008 
Para formular este modelo, é preciso determinar um ponto de partida, ou seja, um produto para o qual a linha já esteja preparada no começo de cada período. Como no caso da indústria de nutrição animal, linha preparada significa linha limpa, e o produto inicial pode ser qualquer um. Alguns testes preliminares mostraram que a escolha de um produto inicial tem grande impacto no resultado do modelo. Desta forma, foi usado o artifício de um produto fantasma $i_{0}$, a partir do qual o tempo de preparação é zero para qualquer outro produto $j\left(s t_{i_{0} j}=0\right)$. Da mesma maneira, para impedir que ocorram preparações a partir de um produto $j$ para o produto $i_{0}$, impõe-se que $s t_{j_{i}}=\infty$. Para não interferir nos resultados do problema, este produto $i_{0}$ também tem tempo de produção e demanda zero, ou seja, $p_{i_{0} t}=0$ e $d_{i_{0} t}=0 \quad \forall t$.

Os índices para formular os modelos são:

$i \quad$ famílias de produtos, $i=1, \ldots, N$

$t \quad$ períodos (e.g., semanas), $t=1, \ldots, T$

onde, $N$ corresponde ao número de famílias e $T$ ao número de períodos.

Os parâmetros dos modelos são:

$C_{t} \quad$ tempo disponível (capacidade) no período $t$

$p_{i} \quad$ tempo necessário para produzir uma unidade da família $i$

$l m_{i} \quad$ tamanho mínimo de um lote da família $i$ (unidades em bateladas)

$h_{i t} \quad$ custo de manter uma unidade de estoque da família $i$ por um período $t$

$c o_{t} \quad$ custo unitário de hora extra no período $t$

$s t_{j i} \quad$ tempo de preparação para mudar da família $j$ para a família $i\left(\operatorname{com} s t_{i i}=0\right)$

$d_{i t} \quad$ demanda da família $i$ no período $t$ (unidades)

$I_{i 0}^{+}, I_{i 0}^{-}$estoque/ backlog inicial da família $i$ no começo do horizonte de planejamento

$u_{t} \quad$ limite máximo de horas extras permitidas no período $t$

$M \quad$ penalização por pedidos pendentes

As variáveis de decisão dos modelos são:

$I_{i t}^{+} \quad$ quantidade em estoque da família $i$ no fim do período $t$

$I_{i t}{ }^{-} \quad$ quantidade pendente (backlog) do produto $i$ no fim do período $t$

$q_{i t} \quad$ tamanho do lote da família $i$ produzido no período $t$. O tamanho do lote é múltiplo do número de bateladas, e, portanto, deve ser uma variável inteira.

$y_{j i t} \quad$ indica se ocorre troca das famílias $j$ para $i$ no período $t\left(y_{j i t}=1\right)$ ou não $\left(y_{j i t}=0\right)$

$O_{t} \quad$ quantidade de horas extras utilizadas no período $t$

Modelo ATSP-períodos: A função objetivo (1) refere-se aos objetivos da empresa, e consiste em minimizar as penalidades por atraso, os custos de estocagem e de horas extras:

$$
\sum_{i=1}^{N} \sum_{t=1}^{T}\left(h_{i t} I_{i t}^{+}+M I_{i t}^{-}\right)+\sum_{t=1}^{T} c o_{t} O_{t}
$$


Diferentemente de Hax \& Candea (1984) e Meyr (2000), a função objetivo (1) não considera custos de preparação, pois, para a empresa estudada, estas preparações consomem apenas tempo, não incorrendo em custos diretos. Ou seja, consideramos que, se existe capacidade disponível, as preparações podem ser realizadas sem custos adicionais. Embora os tempos de preparação não sejam diretamente penalizados na função objetivo, são penalizados indiretamente na utilização de horas extras, quando não há capacidade disponível. Desta forma, a minimização das horas extras na função objetivo inviabiliza preparações desnecessárias. No entanto, do ponto de vista do planejamento da produção, uma solução com menor tempo total de preparação é preferível a uma solução com maior tempo total de preparação. Para diferenciar estas soluções, sem alterar a relação de custos na função objetivo, foi incluída uma pequena penalização para as variáveis de troca $\left(y_{j i t}\right)$ na função objetivo:

$$
\left(\alpha \sum_{j=1}^{N} \sum_{i=1}^{N} s t_{j i} y_{j i t}\right)
$$

onde $\alpha$ representa um número suficientemente pequeno, de forma que o valor da penalização dos tempos de preparação na função objetivo seja irrelevante em relação aos outros termos da função objetivo. Note que as variáveis que representam as trocas $\left(y_{i i t}\right)$ são multiplicadas pelos tempos de preparação $\left(s t_{j i}\right)$. Desta forma, são penalizadas na função objetivo apenas as trocas que envolvem a limpeza da linha. As restrições (3) são de balanceamento de estoques, atrasos, produção e demanda sobre semanas consecutivas:

$$
I_{i t}^{+}-I_{i t}^{-}=I_{i, t-1}^{+}-I_{i, t-1}^{-}+q_{i t}-d_{i t} \quad i=1, \ldots, N ; t=1, \ldots, T
$$

As restrições de capacidade (4) consideram os tempos de preparação, bem como os tempos de produção atuais, e a possibilidade de uma quantidade limitada de horas extras.

$$
\sum_{i=1}^{N} p_{i} q_{i t}+\sum_{j=1}^{N} \sum_{i=1}^{N} s t_{j i} y_{j i t} \leq C_{t}+O_{t} \quad t=1, \ldots, T
$$

Note que a primeira preparação (setup) ocorre a partir do item fantasma $i_{0}$ e então $s t_{i_{0} j}=0$ em todo período $t$. As restrições (5) garantem que a produção de uma família pode ocorrer em um período somente se a linha está preparada para aquele produto.

$$
p_{i} q_{i t} \leq\left(C_{t}+u_{t}\right) \sum_{j=1}^{N} y_{j i t} \quad i=1, \ldots, N ; t=1, \ldots, T
$$

As restrições (6) forçam um lote mínimo e são necessárias porque os tempos de preparação nem sempre satisfazem a desigualdade triangular:

$$
q_{i t} \geq \operatorname{lm}_{i} \sum_{j=1}^{N} y_{j i t} \quad i=1, \ldots, N ; t=1, \ldots, T
$$

Para melhor entender porque as restrições (6) são necessárias, considere uma família $i$ cuja produção contamina a família $k$, a menos que ocorra uma limpeza na linha de produção. Na indústria de nutrição animal, esta limpeza tanto pode ser feita manualmente, interrompendose a produção, ou através da produção de um item 'limpante' $j$, sendo que $s t_{i k}>s t_{i j}+s t_{j k}$. Ou seja, a desigualdade triangular não é válida neste caso. Sem as restrições (6) que impõem 
uma quantidade mínima suficiente de produção do produto $j$ com $q_{j t}>l m_{j}$, permitindo a limpeza adequada do contaminante $i$, um programa ótimo poderia preparar de $i$ para $k$ com $q_{j t}=0$. Note que a desobediência da desigualdade triangular implica que, em certas circunstâncias, uma solução ótima poderia ter mais do que um lote do produto limpante no mesmo período. As pressuposições do modelo de no máximo um lote por período não são válidas em tal situação. Um modelo apropriado para esta situação não é desenvolvido neste artigo, mas apontado como perspectiva para pesquisa futura. As restrições (7) proíbem limpeza da linha entre produtos da mesma família:

$$
y_{i i t}=0 \quad i=1, \ldots, N ; t=1, \ldots, T
$$

Os próximos quatro conjuntos de restrições são relações de dependência próprias do ATSP, e são análogas ao sequenciamento entre os produtos. As restrições (8) permitem uma troca de um produto $j$ para um produto $i$, quaisquer, somente se houve uma troca de $i_{0}$ para um produto $j$ qualquer. Isto garante que $i_{0}$ esteja no começo da sequência em cada período.

$$
\sum_{j=1}^{N} y_{i_{0}, j t} \geq \sum_{j=1}^{N} y_{j i t} \quad i=1, \ldots, N ; i \neq i_{0} ; t=1, \ldots, T
$$

As restrições (9) permitem uma preparação a partir de uma família $j$ (diferente de $i_{0}$ ) somente pode ocorrer se houve uma preparação para esta família $j$.

$$
\sum_{i=1}^{N} y_{i j t} \geq \sum_{k=1}^{N} y_{j k t} \quad j=1, \ldots, N ; j \neq i_{0} ; t=1, \ldots, T
$$

As restrições (10) proíbem mais de uma preparação a partir de uma família $i$, o que significa que em cada período só pode ser produzido um único lote de cada família.

$$
\sum_{j=1}^{N} y_{i j t} \leq 1 \quad i=1, \ldots, N ; t=1, \ldots, T
$$

O sequenciamento a partir das restrições (3)-(10) pode gerar sub-rotas desconexas. Desta forma, são necessárias as restrições (11), que são restrições clássicas de proibição de subrotas (Lawler et al., 1985; Laporte, 1992; Carpaneto et al., 1995; Gutin et al., 2002; Pataki, 2003; Orman \& Williams, 2004; Arenales et al., 2007).

$$
\sum_{i, j \in S r} y_{i j t} \leq|S r|-1 \quad t=1, \ldots, T ; S r \subset P R ; 2 \leq|S r| \leq N-2
$$

onde, $|S r|$ corresponde a cardinalidade de qualquer sub-rota $S_{r}$ formada, e $P R$ é o conjunto de todos os produtos $P R=\{1,2, \ldots, N\}$. As restrições (12) impõem um limite sobre as horas extras trabalhadas, sendo que $u_{t}$ refere-se ao limite máximo de horas extras permitidas pelas leis trabalhistas.

$$
0 \leq O_{t} \leq u_{t} \quad t=1, \ldots, T
$$

Finalmente, as restrições (13), (14) e (15) são restrições de não negatividade e integralidade das variáveis. As restrições (14) proíbem que as variáveis de estoque e atraso sejam negativas:

$$
I_{i t}^{+}, I_{i t}^{-}, \geq 0 \quad i=1, \ldots, N ; t=1, \ldots, T
$$


As restrições (15) garantem que as variáveis de mudança de estado de preparação sejam binárias. E, as restrições (15) impõem que a produção seja um número inteiro de bateladas.

$$
\begin{aligned}
& y_{i j t}=0 \text { ou } 1 \quad i, j=1, \ldots, N ; t=1, \ldots, T \\
& q_{i t} \geq 0 \text { e inteiro } \quad i=1, \ldots, N ; t=1, \ldots, T
\end{aligned}
$$

Conforme discutido antes, dado que os tempos de preparação de algumas famílias de produtos 'limpantes' desobedecem à desigualdade triangular, a formulação anterior não garante produzir uma solução ótima para o problema. Ou seja, pode ocorrer uma situação em que seja mais barato produzir mais de um lote de um mesmo produto para limpar a linha.

Vale ressaltar que, embora seja grande o número de restrições de proibição de sub-rotas, a grande maioria não irá comprometer a solução ótima do modelo. Desta forma, estas restrições são impostas apenas para aquelas sub-rotas que ocorrerem, resolvendo-se uma série de problemas tipo designação (conforme mostrado adiante). Dado que $s t_{i, i_{0}}=\infty \forall i$, as restrições (16) abaixo são redundantes, mas podem ser adicionadas no modelo para tentar acelerar a convergência do método de solução. Elas garantem que não há preparação para a família fantasma $i_{0}$, já preparada no começo de cada período:

$$
y_{i, i_{0} t}=0 \quad i=1, \ldots, N ; t=1, \ldots, T
$$

\subsection{ATSP - Períodos com Sequências Dependentes}

Nesta segunda abordagem pressupõe-se que não existe limpeza semanal, ou seja, a limpeza é realizada conforme existência de risco de contaminação residual. Esta é a estratégia atual da empresa. Desta forma, além do dimensionamento e sequenciamento dos lotes serem simultâneos, deve ser encontrada uma única sequência para todos os períodos. Esta pressuposição torna o problema mais difícil, uma vez que no início de cada período $t \mathrm{o}$ modelo deve 'guardar' o estado de preparação da linha no final do período anterior $(t-1)$ (setup carryover), para que possa determinar qual o primeiro produto da sequência em $t$ que satisfaça as restrições do modelo e minimize a função objetivo. Na abordagem da seção 4.1, este estado de preparação no começo de cada período era tratado como um parâmetro (o produto fantasma $i_{0}$ ). Agora, este estado de preparação deve ser tratado como uma variável. Para formular o modelo é necessária a definição de nova variável:

$z_{i t} \quad$ indica se a família $i$ está preparada no começo do período $t\left(z_{i t}=1\right)$, ou não $\left(z_{i t}=0\right)$. Ou seja, se a família $i$ foi o último produto produzido em $(t-1)$.

\section{Modelo ATSP-carryover:}

A função objetivo (1) e as restrições (3), (4), (7) e (11)-(15) fazem parte do modelo. Entretanto, as demais restrições anteriores devem ser modificadas ou complementadas para formular a dependência da sequência entre os períodos, ou seja, o 'carryover' de um estado de preparação do fim de um período para o começo do próximo. A linha de produção somente pode estar preparada para uma única família no começo do período; isto é garantido pela seguinte restrição:

$$
\sum_{i=1}^{N} z_{i t}=1 \quad t=2, \ldots, T
$$


Observe que esta restrição somente é válida a partir do segundo período, pois no primeiro período a configuração da linha é conhecida; logo, $z_{i 1}$ é um parâmetro. As restrições (5) são substituídas pelas restrições (18), ainda garantindo que a produção de uma família pode ocorrer em um período somente se a linha está preparada. Note que as restrições (18) permitem a produção da família que já esteja preparada no começo do período $t$.

$$
p_{i} q_{i t} \leq\left(C_{t}+u_{t}\right)\left(z_{i t}+\sum_{j=1}^{N} y_{j i t}\right) \quad i=1, \ldots, N ; t=1, \ldots, T
$$

As restrições (6), que forçam a produção de um lote mínimo, não devem ser consideradas para o produto para o qual a linha já está preparada no começo de um período. Ou seja, se $z_{i t}=1$, significa que ocorreu uma preparação para o produto $i$ no período anterior $(t-1) \mathrm{e}$, consequentemente, já ocorreu a produção do lote mínimo. Além do mais, no período $t$, pode não ter demanda para este produto $i$. Desta forma, as restrições (6) precisam ser substituídas pelas restrições (19):

$$
q_{i t} \geq \operatorname{lm}_{i}\left(\sum_{j=1}^{N} y_{j i t}-z_{i t}\right) \quad i=1, \ldots, N ; t=1, \ldots, T
$$

As restrições (8) são substituídas pelas restrições (20), permitindo que um produto $j$ entre na sequência somente se existir uma preparação depois de $j$, ou se $j$ é o produto para a qual a linha está preparada no começo do período próximo período:

$$
\sum_{i=1}^{N} y_{i j t} \leq \sum_{k=1}^{N} y_{j k t}+z_{j, t+1} \quad j=1, \ldots, N ; t=1, \ldots, T-1
$$

As restrições (9) são substituídas pelas restrições (21), permitindo que um produto $k$ entre na sequência depois de $j$ somente se houve uma preparação para $j$, ou se a linha já estava preparada para o produto $j$ no começo do período:

$$
z_{j t}+\sum_{i=1}^{N} y_{i j t} \geq \sum_{k=1}^{N} y_{j k t} \quad j=1, \ldots, N ; t=1, \ldots, T
$$

As restrições (16), que são redundantes no modelo anterior, agora devem ser substituídas pelas restrições (22), que proíbem uma preparação para o produto para o qual a linha já está preparada no começo do período:

$$
1-z_{i t} \geq \sum_{j=1}^{N} y_{j i t} \quad i=1, \ldots, N ; t=1, \ldots, T
$$

As restrições (23) são necessárias para proibir uma nova preparação para o produto já configurado no começo do próximo período:

$$
1-z_{i, t+1} \geq \sum_{j=1}^{N} y_{i j t} \quad i=1, \ldots, N ; t=1, \ldots, T-1
$$

Finalmente, as restrições (24) são necessárias para garantir que ocorra uma preparação depois do produto configurado no começo do período $t$. $\mathrm{O}$ caso raro de não preparação 
em todo aquele período é uma possibilidade alternativa que não é considerada na restrição (24).

$$
z_{i t} \leq \sum_{j=1}^{N} y_{i j t} \quad i=1, \ldots, N ; t=1, \ldots, T
$$

\section{Abordagens de solução para os modelos ATSP}

Se o grupo de restrições que proíbe a formação de sub-rotas (11) for gerado para todas as possíveis sub-rotas $S r$, temos a garantia de obtenção da solução ótima para o problema. Entretanto, dependendo do número de variáveis do problema, a complexidade computacional aumenta, inviabilizando a obtenção da solução em tempo razoável (Orman \& Williams, 2004). Conforme mencionado, a estratégia de solução deste modelo consiste basicamente no seguinte procedimento iterativo: ignora a proibição de sub-rotas; resolve o submodelo de designação; identifica as sub-rotas encontradas nesta solução; e, resolve o submodelo proibindo apenas as sub-rotas identificadas nas soluções anteriores (Lawler et al., 1985; Laporte \& Nobert, 1980; Padberg \& Rinaldi, 1991; Applegate et al., 2003). A aposta desta estratégia é que em poucas iterações é possível encontrar a solução ótima do problema. Este procedimento é mais bem explicado pelo algoritmo que se segue.

\section{Eliminação de Sub-rotas:}

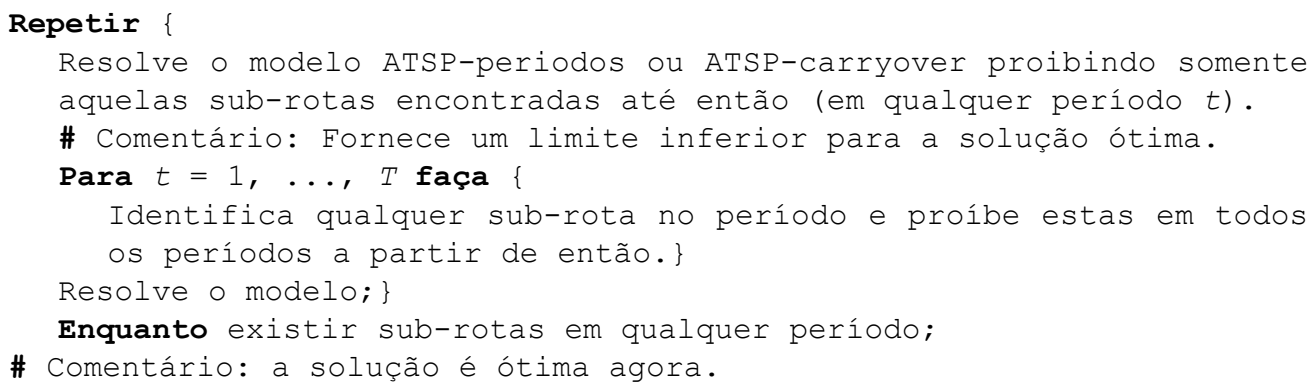

Note que uma eventual solução factível sem sub-rotas não apresenta uma rota circular em cada período, mas uma única sequência, começando com a família já preparada no começo do período e terminando com a última família preparada no período. Um procedimento que pode acelerar a resolução do sequenciamento ATSP consiste em resolver o subproblema de designação e então usar uma heurística de combinação (patching) para juntar as sub-rotas designadas dentro de uma única rota do caixeiro viajante (Karp, 1979; Karp \& Steele, 1985; Frieze \& Dyer, 1990; Pekny \& Miller, 1990; Frieze et al., 1995; Carpaneto et al., 1995; Zhang, 1993; Zhang, 1997; Glover et al., 2001; Gutin \& Zverovich, 2000; Cirasella et al., 2001). O método a seguir é uma extensão do método de eliminação de sub-rotas, onde em cada período as sub-rotas formadas são agrupadas para se obter uma única rota, geralmente gerando uma solução factível a cada iteração. A solução pode desobedecer as restrições (4) se a capacidade for muito apertada com pouca disponibilidade de hora extra. 


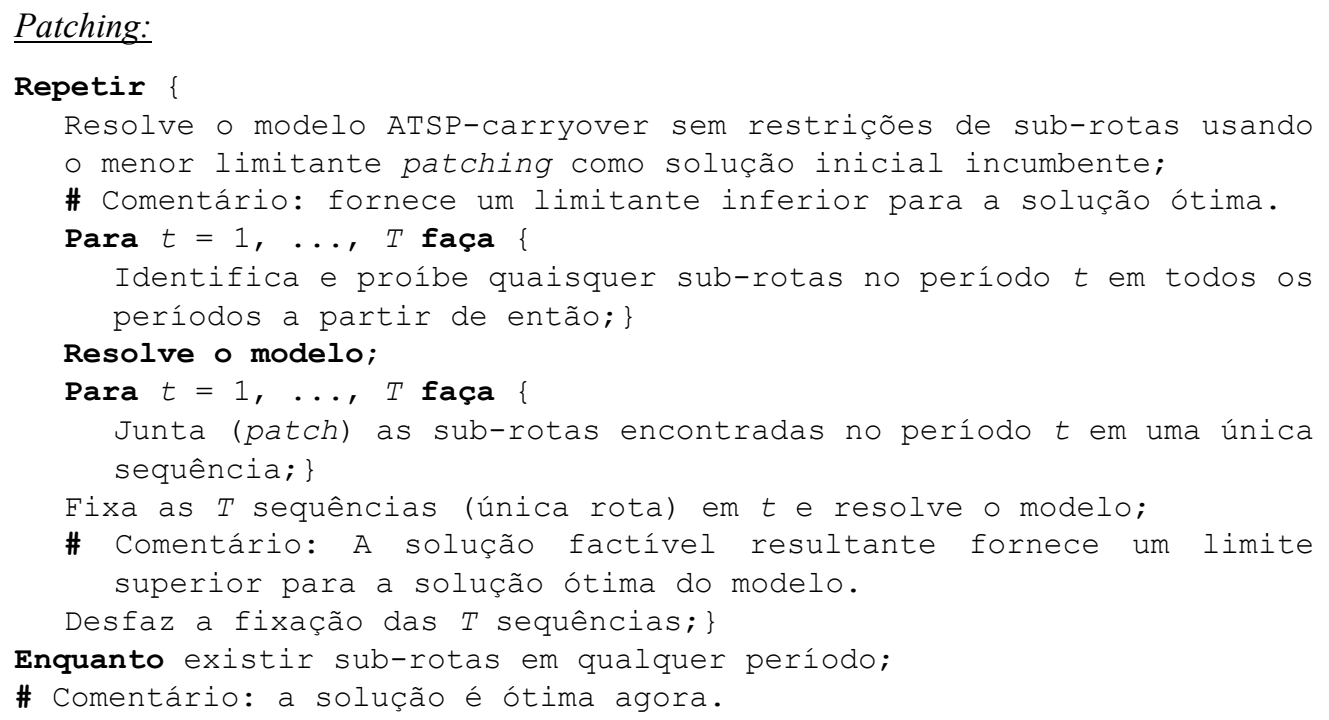

\section{Experimentos computacionais e resultados}

Para validar os modelos e métodos propostos, diversos experimentos computacionais foram realizados utilizando a linguagem de modelagem AMPL com o solver CPLEX versão 9, em um processador Sun V208 Dual Opteron 252 com $1.5 \mathrm{GHz}$ de RAM. Primeiramente, as abordagens foram executadas somente para um período, para testar a consistência da modelagem matemática e computacional, bem como para testar a eficiência do procedimento de proibição de sub-rotas. Os resultados destes experimentos foram bem sucedidos, encontrando-se a solução ótima rapidamente. Desta forma, o próximo passo foi expandir o modelo, incluindo vários períodos e, posteriormente, a dependência entre as sequências dos períodos (carryover). Foram realizados experimentos com a seguinte combinação de modelos/métodos:

i. ATSP-carryover - modelo ATSP - períodos com sequências dependentes / método de eliminação de sub-rotas;

ii. ATSP-patching - modelo ATSP - períodos com sequências dependentes / método patching;

iii. GLSP - modelo GLSP com tempos de preparação e horas extras / método branch and cut (CPLEX) (Toso \& Morabito, 2005);

iv. GLSP-RFvariáveis - modelo GLSP com tempos de preparação e horas extras / método relax-and-fix variáveis (Toso et al., 2007);

v. GLSP-RFperíodos - modelo GLSP com tempos de preparação e horas extras / método relax-and-fix períodos (backward) (Toso et al., 2007);

vi. ATSP-períodos - modelo ATSP - períodos com sequências independentes / método de eliminação de sub-rotas;

vii. GLSP-períodos - modelo GLSP com tempos de preparação, horas extras e períodos com sequências independentes / método branch and cut (CPLEX). 
Para viabilizar a comparação das abordagens ATSP em que os períodos são sequenciados de forma dependente (abordagens (i) e (ii)), com as abordagens GLSP apresentadas em Toso \& Morabito (2005) e Toso et al. (2007) (abordagens (iii), (iv) e (v)), os experimentos GLSP foram refeitos utilizando os mesmos dados e configuração de hardware/software. Para viabilizar a comparação dos resultados da formulação ATSP em que os períodos são sequenciados independentemente (abordagem (vi)), a formulação GLSP foi adaptada de forma que o sequenciamento entre os períodos seja independente, resultando na abordagem (vii). Para isto, é necessário dividir o conjunto de subperíodos $S$ (definido no modelo GLSP) pelo número de períodos $t$, e redefinir as variáveis da seguinte forma:

$q_{\text {ist }} \quad$ tamanho do lote da família $i$ produzido no subperíodo $s$ do período $t$

$x_{i s t} \quad$ indica se a linha está preparada para produzir a família $i$ no subperíodo $s$ do período $t$

$y_{j i s t} \quad$ indica se ocorre troca das famílias $j$ para $i$ no subperíodo $s$ do período $t$

Desta forma, cada período $t$ tem um conjunto $S$ de subperíodos que são sequenciados independentemente, resultando em uma abordagem equivalente a (vi).

\subsection{Estudos com dados reais}

Foram coletados dados reais em meses distintos, de modo que, cada horizonte de planejamento consiste em um mês de produção, subdividido em quatro semanas (t1, t2, t3, t4). Os dois primeiros meses (mesA e mesB) são os mesmos utilizados em Toso \& Morabito (2005) e Toso et al. (2007), e os demais meses (mes $1, \ldots$, mes7) referem-se a dados coletados mais recentemente. Estes dados correspondem: a previsões de demanda de cerca de 200 produtos, agrupados em 26 famílias; a capacidade produtiva em cada período; o tempo disponível para horas extras; os tempos de preparação dependentes que podem ser 0 ou 1,67 horas; os tempos de processamento por produto; estimativas de custos de horas extras e estocagem; e lote mínimo para cada produto. Os estoques iniciais foram descontados da demanda.

A tabela 1 mostra uma breve caracterização destes meses, os dados completos estão em Toso (2008). A segunda coluna mostra o número de famílias de produtos com demanda positiva em cada conjunto de dados. As próximas quatro colunas apresentam a distribuição destas famílias por grau de contaminação. Por exemplo, no mesA, das 18 famílias demandadas, 4 famílias tem grau de contaminação 1, 9 famílias tem grau de contaminação 2, e assim sucessivamente. $\mathrm{O}$ grau de contaminação de cada família indica o quanto ela é crítica para contaminação. Este grau de contaminação foi obtido a partir da matriz de tempos de preparação, calculando-se um tempo médio de preparação necessário depois de cada produto, mais o tempo médio de preparação para cada produto $\left(\sum_{j=1}^{N} s t_{j i}+\sum_{i=1}^{N} s t_{j i}\right) \div 2 N$. Com os resultados, foram estabelecidas as seguintes faixas que correspondem ao grau de contaminação:

- Até 20 minutos --- grau 1 (menos crítico para contaminação);

- Entre 20 e 28 minutos --- grau 2;

- Entre 28 e 35 minutos --- grau 3;

- Acima de 35 minutos --- grau 4 (mais crítico para contaminação). 
Tabela 1 - Caracterização dos dados coletados na empresa.

\begin{tabular}{|c|c|c|c|c|c|c|c|c|c|c|}
\hline \multirow{2}{*}{ Dados } & \multirow{2}{*}{$\mathrm{N}^{\circ}$ famílias } & \multicolumn{6}{|c|}{ Distribuição/ Grau de Contaminação } & \multicolumn{5}{|c|}{ Capacidade Necessária (\%) } \\
\cline { 3 - 10 } & & 1 & 2 & 3 & 4 & $t=1$ & $t=2$ & $t=3$ & $t=4$ & média \\
\hline mês A & 18 & 4 & 9 & 3 & 2 & 90 & 88 & 96 & 117 & 97,75 \\
mês B & 15 & 2 & 7 & 2 & 1 & 97 & 104 & 126 & 91 & 104,5 \\
mês 1 & 20 & 5 & 11 & 3 & 1 & 87 & 85 & 103 & 116 & 97,75 \\
mês 2 & 21 & 4 & 10 & 5 & 2 & 78 & 102 & 91 & 129 & 100 \\
mês 3 & 18 & 5 & 10 & 2 & 1 & 93 & 92 & 108 & 94 & 96,75 \\
mês 4 & 19 & 5 & 10 & 2 & 1 & 73 & 76 & 88 & 104 & 85,25 \\
mês 5 & 22 & 5 & 9 & 5 & 2 & 81 & 96 & 104 & 135 & 104 \\
mês 6 & 18 & 5 & 10 & 2 & 1 & 87 & 92 & 95 & 134 & 102 \\
mês 7 & 20 & 5 & 11 & 2 & 2 & 84 & 105 & 94 & 132 & 103,75 \\
\hline
\end{tabular}

Desta forma, um produto mais crítico para contaminação tanto pode significar um produto muito contaminante, depois do qual a linha quase sempre precisa ser limpa, quanto um produto muito suscetível à contaminação. Note que a quantidade destes itens mais críticos (graus 3 e 4) é relativamente pequena; a grande maioria dos produtos demandados são menos críticos para contaminação. Isto significa que é relativamente fácil encontrar sequências em que são necessárias poucas interrupções na produção para preparação da linha. As últimas quatro colunas da tabela 1 estão relacionadas à porcentagem de capacidade necessária para produção da demanda em cada período $t$, ou seja, antes de considerar os tempos necessários para preparação. Em algumas semanas a capacidade necessária para produção é maior que $100 \%$, ou seja, não é possível produzir a demanda sem a utilização de horas extras. Note que existe uma tendência em acumular a demanda nos últimos períodos (padrão de demanda da empresa).

\subsubsection{Períodos com Sequências Independentes}

A tabela 2 apresenta os resultados obtidos para as abordagens onde a sequência considera períodos independentes (abordagens (vi) e (vii)). Para cada modelo são apresentados: o valor da função objetivo; o tempo computacional em segundos em que cada solução foi obtida, uma vez que todos os experimentos foram limitados em uma hora (3600 segundos); e, o gap de otimalidade.

Note na tabela 2 que, para quase todos os experimentos realizados, o valor da função objetivo é o mesmo, entretanto, o tempo computacional varia bastante. Exceto para o exemplar mes5, a estratégia ATSP teve melhor desempenho computacional (destacados na tabela). Em média o ATSP é cerca de 17\% mais rápido que o GLSP. Uma diferença importante entre estas abordagens é que, dada a limitação do tempo de 3600 segundos, a abordagem ATSP sempre converge para a solução ótima dentro deste limite (exceto para o mes7, em que este limite foi atingido), enquanto que as soluções GLSP, mesmo depois de uma hora de execução, não têm garantia de otimalidade. 
Tabela 2 - Resultados dos modelos / métodos para a abordagem Sequências Independentes.

\begin{tabular}{|c|ccc|ccccc|}
\hline & \multicolumn{3}{|c|}{ GLSP-períodos } & \multicolumn{5}{c|}{ ATSP-períodos } \\
& f.o. & tempo & gap $(\%)$ & f.o. & tempo & iterações & sub-rotas & gap (\%) \\
\cline { 2 - 9 } mesA & 3028 & $2860 / 3600$ & 3,28 & $\mathbf{3 0 2 8}$ & $\mathbf{3 3 6}$ & 60 & 240 & 0 \\
mesB & 15425 & $779 / 3600$ & 0,18 & $\mathbf{1 5 4 2 5}$ & $\mathbf{9}$ & 28 & 86 & 0 \\
mes1 & 4029 & $\mathbf{7 0 6} / 3600$ & 1,08 & $\mathbf{4 0 2 3}$ & 3542 & 118 & 587 & 0 \\
mes2 & 7655 & $2531 / 3600$ & 4,69 & $\mathbf{7 6 5 5}$ & $\mathbf{2 0 1 1}$ & 194 & 916 & 0 \\
mes3 & 3207 & $968 / 3600$ & 0,99 & $\mathbf{3 2 0 7}$ & $\mathbf{4 6 0}$ & 160 & 686 & 0 \\
mes4 & 3378 & $234 / 3600$ & 5,31 & $\mathbf{3 3 7 8}$ & $\mathbf{1 0 4}$ & 97 & 419 & 0 \\
mes5 & $\mathbf{1 3 0 5 6}$ & $\mathbf{2 8} / 3600$ & 0 & 13056 & 43 & 113 & 486 & 0 \\
mes6 & 9611 & $790 / 3600$ & 0,23 & $\mathbf{9 6 1 1}$ & $\mathbf{3 9 9}$ & 14 & 76 & 0 \\
mes7 & 9088 & $\mathbf{2 5 7 9} / 3600$ & 6,09 & $\mathbf{9 0 7 6}$ & 3600 & 4 & 20 & 0,22 \\
média & 7609 & $1275 / 3600$ & 2,43 & $\mathbf{7 6 0 7}$ & 1167 & 88 & 391 & 0 \\
\hline
\end{tabular}

Conforme esperado, os experimentos com as abordagens GLSP-períodos e ATSP-períodos mostraram que, como consequência do sequenciamento independente, os produtos mais contaminantes são alocados ao final de cada período, não considerando que deve existir uma preparação para iniciar a produção no próximo período. Desta forma, é importante a pressuposição de que é necessário fazer uma limpeza ao final de cada período. A tabela 3 apresenta um resumo dos programas de produção ótimos resultantes da abordagem com sequenciamento independente entre os períodos. Para todos os conjuntos de dados são apresentados: a capacidade utilizada em cada período, as horas extras necessárias e o número de preparações ou limpezas necessárias para evitar a contaminação residual.

Tabela 3 - Resumo dos Programas de Produção Ótimos - Sequências Independentes.

\begin{tabular}{|c|c|c|c|c|c|c|c|c|c|}
\hline \multirow{2}{*}{ Dados } & \multicolumn{4}{|c|}{ Capacidade utilizada } & \multicolumn{4}{c|}{ Horas Extras } & \multirow{2}{*}{ Preparações } \\
\cline { 2 - 8 } & $\mathrm{t} 1$ & $\mathrm{t} 2$ & $\mathrm{t} 3$ & $\mathrm{t} 4$ & $\mathrm{t} 1$ & $\mathrm{t} 2$ & $\mathrm{t} 3$ & $\mathrm{t} 4$ & \\
\hline mesA & 90.8 & 100 & 100 & 100 & 0 & 0 & 0 & 0 & 0 \\
mesB & 100 & 102.2 & 125 & 90.9 & 0 & 1.4 & 16 & 0 & 0 \\
mes1 & 91 & 100 & 100 & 100.2 & 0 & 0 & 0 & 0.1 & $1 \mathrm{em} \mathrm{t3}$ \\
mes2 & 100 & 100 & 100 & 102.9 & 0 & 0 & 0 & 1.5 & $1 \mathrm{em} \mathrm{t}$ \\
mes3 & 93.5 & 99.8 & 100 & 94,3 & 0 & 0 & 0 & 0 & 0 \\
mes4 & 73.1 & 75.6 & 91.5 & 99.8 & 0 & 0 & 0 & 0 & 0 \\
mes5 & 100 & 100 & 100 & 115.8 & 0 & 0 & 0 & 8.2 & 0 \\
mes6 & 99.8 & 100 & 100 & 107.9 & 0 & 0 & 0 & 4.1 & 0 \\
mes7 & 99.7 & 100 & 100 & 119 & 0 & 0 & 0 & 8 & 1 em t1 \\
\hline
\end{tabular}

Observe nas tabelas 1 e 3 que as abordagens antecipam a produção, evitando sempre que possível a alternativa de utilização de horas extras. Entretanto, conforme discutido adiante, a estratégia adotada pela empresa é acompanhar a demanda, recorrendo quando necessário às horas extras. Como esta abordagem pressupõe a realização de uma limpeza ao final de cada 
período, são necessárias pelo menos $2,6 \%$ da capacidade produtiva para esta tarefa. No entanto, não existe capacidade disponível em todos os períodos (tabela 3). Desta forma, para estes conjuntos de dados analisados, esta estratégia implica necessariamente em um custo extra.

\subsubsection{Períodos com Sequências Dependentes}

A tabela 4 apresenta os resultados obtidos para as abordagens em que o sequenciamento considera períodos dependentes (abordagens (i)-(v)). Nesta tabela, para cada conjunto de dados (mês) existem duas linhas: a superior corresponde ao valor da função objetivo e a inferior corresponde ao tempo computacional (em segundos) no qual cada solução foi obtida. Os experimentos GLSP foram limitados em 3600 segundos, enquanto que os experimentos ATSP são resolvidos até a otimalidade (note que apenas no mes5 a abordagem ATSPcarryover excede ligeiramente este limite -3609 segundos).

Tabela 4 - Resultados dos modelos / métodos para a abordagem Sequências Dependentes.

\begin{tabular}{|c|c|c|c|c|c|}
\hline & GLSP & $\begin{array}{c}\text { GLSP- } \\
\text { RFvariáveis }\end{array}$ & $\begin{array}{c}\text { GLSP- } \\
\text { RFperiodos }\end{array}$ & $\begin{array}{c}\text { ATSP- } \\
\text { carryover }\end{array}$ & $\begin{array}{l}\text { ATSP- } \\
\text { patching }\end{array}$ \\
\hline \multirow{2}{*}{ mesA } & 3519 & 3453 & 3445 & 3445 & 3445 \\
\hline & $(360 / 3600)$ & $(1273 / 3600)$ & $(415$ / 3600) & (2374) & (9) \\
\hline \multirow{2}{*}{ mesB } & 16616 & 16616 & 16616 & 16616 & 16616 \\
\hline & $(157 / 3600)$ & $(150 / 3600)$ & $(511 / 3600)$ & $(0,93)$ & $(0,35)$ \\
\hline \multirow{2}{*}{ mes1 } & 4312 & 4312 & 4312 & 4312 & 4312 \\
\hline & $(339 / 3600)$ & $(132$ / 3600) & $(115 / 3600)$ & (643) & (472) \\
\hline \multirow{2}{*}{ mes2 } & 8176 & 8176 & 8176 & 8176 & 8176 \\
\hline & $(438 / 3600)$ & $(258$ / 3600) & $(65 / 3600)$ & (1098) & (196) \\
\hline \multirow{2}{*}{ mes3 } & 4328 & 4328 & 4589 & 4281 & 4281 \\
\hline & $(1049 / 3600)$ & $(240 / 3600)$ & $(243 / 3600)$ & (273) & (279) \\
\hline \multirow{2}{*}{ mes4 } & 3378 & 3378 & 3378 & 3378 & 3378 \\
\hline & $(2854 / 3600)$ & $(183$ / 3600) & $(2150 / 3600)$ & (264) & (1) \\
\hline \multirow{2}{*}{ mes5 } & 13510 & 13510 & 13510 & 13510 & 13510 \\
\hline & $(775 / 3600)$ & $(117 / 3600)$ & $(341 / 3600)$ & $(3600)$ & (4) \\
\hline \multirow{2}{*}{ mes6 } & 10019 & 10019 & 10019 & 10019 & 10019 \\
\hline & $(345 / 3600)$ & $(128 / 3600)$ & $(227 / 3600)$ & (1082) & (2) \\
\hline \multirow{2}{*}{ mes7 } & 11597 & 10726 & 10851 & 10237 & 10237 \\
\hline & $(2998 / 3600)$ & $(484$ / 3600) & $(1506 / 3600)$ & (848) & (7) \\
\hline \multirow{2}{*}{ média } & 8384 & 8280 & 8322 & 8219 & 8219 \\
\hline & $1035 / 3600$ & $(329 / 3600)$ & $(619 / 3600)$ & (1131) & (108) \\
\hline
\end{tabular}

Observe nestes experimentos que as diferenças em relação aos valores obtidos para a função objetivo não são significantes de uma estratégia para outra, sendo que para os dados mesB, mes1, mes2, mes4, mes5 e mes6, a solução obtida é a mesma. Por outro lado, os tempos computacionais são bem distintos. Em geral a estratégia ATSP-patching é bem mais rápida, 
obtendo a solução ótima em menor tempo em seis dos nove experimentos. A segunda estratégia em menor tempo computacional é a abordagem GLSP-RFvariáveis, com tempo médio de resolução de 329 segundos. No entanto, as soluções GLSP que algumas vezes são obtidas depois de pouco tempo computacional, não são melhoradas ou provadas como ótimas dentro de uma hora de processamento. Neste sentido, a abordagem ATSP-carryover é melhor que a abordagem GLSP-RFvariáveis nestes experimentos.

A tabela 5 apresenta um resumo do programa de produção ótimo resultante da abordagem com sequenciamento dependente entre os períodos. Conforme esperado, quando a capacidade é restritiva, o número de preparações tende a ser minimizado pelo modelo. As abordagens, sempre que possível, agrupam a demanda dos produtos com maior grau de contaminação em lotes maiores, diminuindo a necessidade de limpezas adicionais. No entanto, esta decisão depende de vários fatores: a distribuição da demanda entre os períodos; a capacidade disponível para agrupar lotes de famílias de produtos com maior grau de contaminação, bem como a viabilidade de custos resultante (estocagem); e, a quantidade de famílias críticas para o sequenciamento. Em todos os programas de produção resultantes, a demanda é atendida sem atrasos. A análise detalhada dos programas de produção obtidos nos experimentos realizados mostra que existem algumas características comuns no sequenciamento, como, por exemplo, o produto final na sequência de um determinado período geralmente é o produto inicial na sequência do próximo período.

Tabela 5 - Resumo dos Programas de Produção Ótimos - Sequências Dependentes.

\begin{tabular}{|c|c|c|c|c|c|c|c|c|c|c|c|c|}
\hline \multirow{2}{*}{ Dados } & \multicolumn{4}{|c|}{ Capacidade utilizada } & \multicolumn{4}{|c|}{ Horas Extras } & \multicolumn{3}{c|}{ Preparação } \\
\cline { 2 - 12 } & $\mathrm{t} 1$ & $\mathrm{t} 2$ & $\mathrm{t} 3$ & $\mathrm{t} 4$ & $\mathrm{t} 1$ & $\mathrm{t} 2$ & $\mathrm{t} 3$ & $\mathrm{t} 4$ & $\mathrm{t} 1$ & $\mathrm{t} 2$ & $\mathrm{t} 3$ & $\mathrm{t} 4$ \\
\hline mesA & 96 & 100 & 100 & 100 & 0 & 0 & 0 & 0 & 1 & 1 & 0 & 0 \\
mesB & 100.1 & 104.7 & 125 & 93.5 & 0.07 & 0.27 & 0 & 9.2 & 1 & 0 & 0 & 1 \\
mes1 & 94.2 & 99.9 & 100 & 100.2 & 0 & 0 & 0 & 5.7 & 0 & 1 & 0 & 0 \\
mes2 & 100.1 & 100 & 100 & 106 & 0 & 0 & 0 & 0.1 & 2 & 0 & 0 & 0 \\
mes3 & 97 & 99.4 & 100 & 97.4 & 0 & 0 & 0 & 10 & 1 & 0 & 0 & 1 \\
mes4 & 73.1 & 79.1 & 94.9 & 99.8 & 0.04 & 0 & 0 & 3.1 & 0 & 1 & 1 & 0 \\
mes5 & 99.8 & 100 & 100 & 119.2 & 0 & 0 & 0 & 0 & 1 & 0 & 0 & 0 \\
mes6 & 99.9 & 100 & 100 & 111 & 0 & 0 & 0 & 0 & 1 & 0 & 0 & 0 \\
mes7 & 100.2 & 100.6 & 100 & 121.9 & 0.07 & 3 & 16 & 0 & 1 & 1 & 0 & 0 \\
\hline
\end{tabular}

\subsubsection{Comparação Sequências Independentes e Dependentes}

Para comparar as estratégias 'Sequências Independentes' e 'Sequências Dependentes', além dos experimentos apresentados acima, foi realizado um experimento com os modelos 'Sequências Independentes' com redução da capacidade disponível para produção no tempo equivalente a uma preparação em cada período (1,67 horas). A figura 1 apresenta uma comparação entre os valores médios de: capacidade utilizada; número de horas extras; e número de preparações dos programas de produção resultantes. Para a estratégia sequências independentes com redução de capacidade, os valores da função objetivo sempre resultam em custos maiores, embora a limpeza semanal reduza o número de preparações necessárias, e a redução de capacidade nos períodos implica no aumento dos custos de horas extras e 
estocagem. Vale lembrar que a comparação entre estas estratégias não é direta, pois envolvem diferentes pressupostos. Por exemplo, na estratégia sequências independentes sem redução de capacidade, não foram considerados os prováveis custos extras da limpeza semanal. No entanto, os resultados destes experimentos mostram que, se a empresa adotar a limpeza semanal dentro do período de produção, o impacto nos custos é significativo. Além disso, os custos de oportunidade devem ser adequadamente avaliados, pois a limpeza consome grande parte da capacidade produtiva.

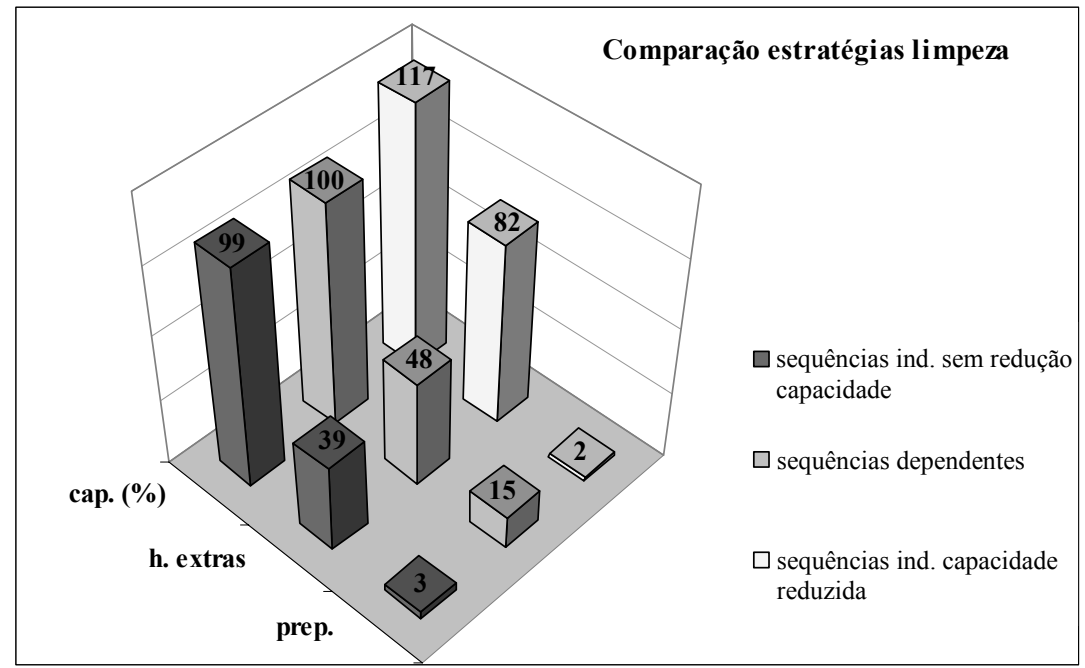

Figura 1 - Comparação dos programas de produção resultantes para as estratégias de limpeza.

O programa de produção da empresa é feito com base na estratégia de acompanhar a demanda a cada período, o que resulta em custos substancialmente maiores. Conforme mostrado em Toso \& Morabito (2005), para os dados referentes ao mesA, o programa de produção da empresa resulta em um custo cerca de $70 \%$ maior em relação às soluções geradas pelo GLSP. Isto ocorre porque, além de antecipar a produção nos períodos onde existe capacidade ociosa, os modelos encontram sequências de produção melhores, com menos preparações. Cabe salientar que o programa de produção da empresa é cauteloso em relação ao aumento de demanda previsto para o final do mês, ou seja, a programação da empresa aposta que este aumento não vai ocorrer. Se isto acontecer de fato, a estratégia de produção da empresa pode ser melhor do que parece. Entretanto, esta cautela da empresa em relação às incertezas da demanda pode ser contemplada no modelo por meio de análise de sensibilidade, alterando-se os parâmetros de demanda por valores menos otimistas e executando-se o programa para diversos cenários.

\subsection{Estudos com Dados Aleatórios}

Para ampliar a análise dos modelos e métodos propostos, foram realizados diversos experimentos com perturbações nos dados fornecidos, bem como, com geração de dados aleatórios. Nestes experimentos foram alterados diversos parâmetros. A tabela 6 apresenta uma síntese dos resultados dos seguintes experimentos: redução da capacidade; geração de 
novos padrões de demanda (aleatórios); e, geração de novos dados para a matriz de tempos de preparação. Por motivo de limitação de espaço, nesta seção discutimos resumidamente os principais resultados obtidos - para mais detalhes destes e outros resultados, os leitores podem consultar Toso (2008).

Tabela 6 - Porcentagem de soluções factíveis em cada experimento.

\begin{tabular}{|c|c|c|c|c|c|c|c|c|}
\hline & \multicolumn{5}{|c|}{ SEQUENNCIAS DEPENDENTES } & \multicolumn{2}{|c|}{$\begin{array}{c}\text { SEQUÊNCIAS } \\
\text { INDEPENDENTES }\end{array}$} \\
\hline & & GLSP & $\begin{array}{l}\text { GLSP RF } \\
\text { variáveis }\end{array}$ & $\begin{array}{l}\text { GLSP RF } \\
\text { períodos }\end{array}$ & $\begin{array}{c}\text { ATSP } \\
\text { carryover }\end{array}$ & $\begin{array}{c}\text { ATSP } \\
\text { patching }\end{array}$ & $\begin{array}{c}\text { GLSP } \\
\text { períodos }\end{array}$ & $\begin{array}{c}\text { ATSP } \\
\text { períodos }\end{array}$ \\
\hline \multicolumn{2}{|c|}{ Dados reais } & 100 & 100 & 100 & 100 & 100 & 100 & 100 \\
\hline \multirow{2}{*}{$\begin{array}{l}\text { Redução } \\
\text { capacidade }\end{array}$} & $-10 \%$ & 100 & 100 & 100 & 100 & 100 & 100 & 100 \\
\hline & $-20 \%$ & 100 & 100 & 100 & 100 & 100 & 100 & 100 \\
\hline \multirow{3}{*}{$\begin{array}{l}\text { Alteração } \\
\text { demanda }\end{array}$} & a & 100 & 100 & 100 & 100 & 100 & 100 & 100 \\
\hline & $\mathrm{b}$ & 100 & 100 & 80 & 100 & 100 & 80 & 100 \\
\hline & $\mathrm{c}$ & 70 & 80 & 70 & 80 & 80 & 80 & 80 \\
\hline \multirow{3}{*}{$\begin{array}{l}\text { Alteração } \\
\text { tempos de } \\
\text { preparação }\end{array}$} & $d$ & 33 & 0 & 0 & 44 & 100 & 11 & 78 \\
\hline & e & 11 & 0 & 0 & 89 & 100 & 0 & 100 \\
\hline & $\mathrm{f}$ & 100 & 89 & 22 & 100 & 100 & 22 & 100 \\
\hline \multicolumn{2}{|l|}{ Média } & 88 & 85 & 80 & 77 & 99 & 81 & 97 \\
\hline
\end{tabular}

Com o objetivo de verificar o desempenho dos modelos e métodos diante de cenários de capacidade mais restritiva, foram realizados experimentos diminuindo-se a capacidade produtiva em 10 e $20 \%$ (Tabela 6 ), de maneira que sua distribuição nos períodos não seja alterada, isto é, em todos os períodos a capacidade é igualmente reduzida. Para todos os outros parâmetros foram mantidos os valores coletados na empresa. Experimentos que alteram a distribuição de capacidade ao longo dos períodos são contemplados nos experimentos com geração de demandas aleatórias. Como esperado, a redução da capacidade tem impacto direto nos custos, principalmente nos custos de horas extras, já que comprometem o tempo para produção em todos os períodos. Em relação ao desempenho das abordagens testadas, observamos nestes experimentos que os cenários de capacidade mais restritiva não alteram significativamente o desempenho das abordagens. Como nos experimentos com dados reais, os melhores resultados foram obtidos pelas abordagens ATSP.

A demanda por produtos na indústria de suplementos para nutrição animal tem algumas peculiaridades, por exemplo, o mix de produtos e a quantidade demandada variam muito de um período para o outro. Desta forma, o objetivo nestes experimentos é testar diferentes configurações de demanda. Foram realizados três testes: a) concentração da demanda no final do último período do horizonte de planejamento $(t=4)$; b) geração de demanda aleatória entre os valores mínimos e máximos da demanda real para cada produto; c) geração de dados de demanda aleatórios dentro do mesmo intervalo para todos os produtos, tornando as instâncias mais difíceis (Tabela 6).

Como efeito da concentração da demanda, os custos de estocagem aumentam substancialmente. Em relação à qualidade das soluções obtidas, todas as abordagens encontram a 
solução ótima para todas as instâncias; no entanto, as soluções das abordagens GLSP não têm garantia de otimalidade. Nos experimentos com geração de demanda aleatória, dentro do limite mínimo e máximo das demandas reais, o comportamento das abordagens não difere muito em relação aos experimentos com dados reais. Os maiores impactos foram observados nas abordagens GLSP RF períodos e GLSP períodos (Tabela 6).

A geração de dados aleatórios de demanda para todos os produtos dentro do mesmo intervalo resulta em instâncias mais difíceis, na medida em que a probabilidade de ocorrência de demanda por produtos mais contaminantes é a mesma que a de produtos menos contaminantes. Neste sentido, observamos que as soluções geradas por estes experimentos apresentam um maior tempo médio de preparações. Nestes experimentos, $20 \%$ das instâncias geradas não foram resolvidas por nenhuma abordagem. Entre as instâncias resolvidas, observamos que: para a estratégia sequências dependentes, as abordagens ATSP são melhores, obtendo as soluções ótimas em quase todas as instâncias geradas; a abordagem GLSP RF variáveis apresenta bons resultados, encontrando soluções muito próximas dos valores ótimos; para a estratégia sequências independentes, as abordagens ATSP e GLSP encontram valores ótimos em todas as instâncias.

Em geral, os resultados dos experimentos com alteração na demanda mostram que as abordagens GLSP são mais sensíveis à geração de dados aleatórios. Quanto às abordagens ATSP, embora a qualidade das soluções seja menos comprometida do que da abordagem GLSP, o tempo de execução computacional, principalmente para a abordagem ATSPcarryover, é maior do que nos experimentos com dados reais. As melhores abordagens de solução para as sequências dependentes e sequências independentes são: ATSP-patching e ATSP-períodos, respectivamente.

Nos experimentos com alterações na matriz de tempos de preparação foram realizados os seguintes testes: d) inclusão dos tempos de preparação pequenos (inicialmente desprezados) na matriz real; e) geração de matriz de tempos de preparação simétrica, com desigualdade triangular válida; f) geração de matriz de tempos de preparação menos esparsa (Tabela 6). Nos experimentos (d) e (e), as soluções encontradas por todas as abordagens pioram significativamente; nenhuma das abordagens encontra a solução ótima para nenhuma das instâncias dentro do limite de tempo. Os melhores resultados são obtidos pelas abordagens ATSP-patching e ATSP-períodos.

A matriz de tempos de preparação fornecida pela empresa é bastante esparsa, apenas $25 \%$ dos tempos de preparação são positivos. Entretanto, nos experimentos com dados reais, nem todas as abordagens testadas encontram soluções com garantia de otimalidade para todas as instâncias. Quando geramos valores para a matriz de preparação 0 ou 1,67 horas, a partir de valores uniformemente distribuídos entre 0 e 1 , o valor esperado de valores positivos é $50 \%$. No entanto, nos experimentos realizados com esta matriz de preparação todas as abordagens encontram a solução ótima para todas as instâncias.

Os experimentos com alterações na matriz de tempos de preparação tiveram maior impacto no desempenho das abordagens testadas, principalmente nas abordagens GLSP. Para muitas instâncias, as abordagens GLSP não encontram ao menos soluções factíveis. O desempenho da abordagem ATSP-carryover também é significativamente afetado. Para algumas instâncias, também não são encontradas soluções factíveis, dentro do limite de tempo. Novamente, as abordagens com melhor desempenho foram ATSP-patching e ATSPperíodos. 
Em geral, nos experimentos com alteração nos dados fornecidos pela empresa, observamos que a característica de maior impacto no desempenho dos modelos propostos é a estrutura dos tempos de preparação. O problema integrado de dimensionamento e sequenciamento de lotes de produção na indústria de suplementos possui uma estrutura particular de tempos de preparação que o diferencia dos problemas geralmente tratados na literatura. A matriz de tempos de preparação desta indústria não obedece à desigualdade triangular, é assimétrica e, no caso da empresa estudo de caso, também é esparsa.

\section{Conclusões}

Neste artigo, o problema de dimensionamento e sequenciamento de lotes na indústria de nutrição animal foi reformulado, usando a abordagem ATSP para representar as decisões de sequenciamento de lotes. Foram utilizados dois modelos para descrever o problema, baseados em diferentes estratégias para a limpeza da linha de produção. O modelo ATSP 'Sequências Independentes' faz o sequenciamento dos períodos independentemente, mantendo as decisões de dimensionamento integradas. O modelo ATSP 'Sequências Dependentes' resolve o sequenciamento de todos os períodos integrados, usando uma variável para conservar o estado de preparação entre os períodos. Para resolver estes modelos foram aplicados dois métodos: eliminação de sub-rotas e uma combinação de eliminação de sub-rotas com a heurística patching.

O modelo GLSP para ração animal também foi adaptado para a estratégia da empresa de sequenciar os períodos de forma independente. Desta forma, foi possível realizar experimentos e comparar as abordagens GLSP e ATSP para as diferentes estratégias.

Nos experimentos com dados reais, as abordagens GLSP fornecem soluções ótimas ou próximas do ótimo, mas sem a garantia de otimalidade, pois depois do limite de tempo de uma hora de processamento existe uma distância destas soluções em relação ao limitante inferior (gap de otimalidade), que varia de cerca de 3 a $20 \%$. As abordagens ATSP foram mais bem sucedidas, mais rápidas na maior parte dos experimentos, e com a vantagem de provar a otimalidade das soluções dentro de uma hora de execução.

Nos experimentos com alteração nos dados fornecidos pela empresa, observamos que a característica de maior impacto no desempenho dos modelos propostos é a estrutura dos tempos de preparação. O problema integrado de dimensionamento e sequenciamento de lotes de produção na indústria de suplementos possui uma estrutura particular de tempos de preparação que o diferencia dos problemas tratados na literatura.

Considerando todos os experimentos realizados, podemos afirmar que a melhor estratégia para abordar o problema foi o modelo ATSP - períodos com sequências dependentes, resolvido com o método combinado de eliminação de sub-rotas e patching. Os tempos computacionais nesta estratégia são aceitáveis considerando as decisões envolvidas na prática, possibilitando a geração e análise de diferentes cenários de planejamento, e respostas rápidas aos clientes sobre a inclusão / cancelamento de pedidos em carteira; desta maneira, fornecendo uma importante ferramenta para o planejamento e programação da produção na empresa. Uma perspectiva interessante para pesquisa futura é estender as abordagens apresentadas para tratar as linhas de produção dois estágios da indústria de nutrição animal, que correspondem à fabricação de rações extrusadas, peletizadas e trituradas. 


\section{Agradecimentos}

Os autores agradecem aos revisores anônimos pelos úteis comentários e sugestões. Esta pesquisa contou com apoio do CNPq (processo 522973/95-4) e da agência inglesa EPSRC (Engineering and Physical Sciences Research Council) (referência EP/D000483/1).

\section{Referências Bibliográficas}

(1) Allahverdi, A.; Gupta, J.N.D. \& Aldowaisan, T. (1999). A Review of Scheduling Research Involving Setup Considerations. Omega International Journal of Management Science, 27, 219-239.

(2) Applegate, D.; Bixby, R.; Chvatal, V. \& Cook, W. (2003). Implementing the DantzigFulkerson-Johnson Algorithm for Large Traveling Salesman Problems. Mathematical Programming, 97, 91-153.

(3) Araujo, S.A. \& Arenales, M.N. (2003). Problema de Dimensionamento de Lotes Monoestágio com Restrição de Capacidade: Modelagem, Método de Resolução e Resultados Computacionais. Pesquisa Operacional, 23(3), 403-420.

(4) Araujo, S.A.; Arenales, M.N. \& Clark, A.R. (2004). Dimensionamento de lotes e programação do forno numa fundição de pequeno porte. Gestão \& Produção, 11(2), $165-176$

(5) Arenales, M.N.; Armentano, V.A.; Morabito, R. \& Yanasse, H.H. (2007). Pesquisa Operacional. 1a. ed. Campus / Elsevier, Rio de Janeiro, v.1. 523 p.

(6) Armentano, V.A.; França, P.M. \& Toledo, F.M.B. (1999). A network flow model for the capacitated lot-sizing problem. Omega, 27, 275-284.

(7) Askin, R. \& Standridge, C. (1993). Modeling and analysis of manufacturing systems. John Wiley \& Sons, NY.

(8) Blazewicz, J.; Domschke, W. \& Pesch, E. (1996) The job shop scheduling problem: Conventional and new solution techniques. European Journal of Operational Research, 93(1), 1-33(33).

(9) Brito, A.B. \& Stringhini, J.H. (2002). Site - Nutriline <http://nutrimail.vila.bol.com.br> (web page). Acessado em: 23/07/2006.

(10) Buriol, L.; Franca, P.M. \& Moscato, P. (2003). A new memetic algorithm for the asymmetric traveling salesman problem. Relatório Técnico, Faculdade de Engenharia Elétrica, Universidade Estadual de Campinas.

(11) Carpaneto, G.; Dell'Amico, M. \& Toth, P. (1995). Exact solution of large scale asymmetric travelling salesman problems. ACM Transactions on Mathematical Software, 21(4), 394-409.

(12) Cirasella, J.; Johnson, D.S.; McGeoch, L.A. \& Zhang, W. (2001). The asymmetric traveling salesman problem: Algorithms, instance generators, and tests. Springer Lecture Notes in Computer Science, 2153, 32-59.

(13) Clark, A.R. \& Clark, S.J. (2000). Rolling-horizon lot-sizing when set-up times are sequence-dependent. International Journal of Production Research, 38, 2287-2307. 
(14) Clark, A.R. (2003). Optimization Approximations for Capacity Constrained Material Requirement Planning. Internal Research Report. MS-20020-2. Bristol: University of the West of England.

(15) Drexl, A. \& Haase, K. (1995). Proportional Lot sizing and Scheduling. International Journal of Production Economics, 40, 73-87.

(16) Drexl, A. \& Kimms, A. (1997). Lot sizing and Scheduling - Survey and extensions. European Journal of Operational Research, 99(2), 221-235.

(17) Ferreira, D.; Morabito, R. \& Rangel, S. (2008). Um modelo de otimização inteira mista e heurísticas relax and fix para a programação da produção de fábricas de refrigerantes de pequeno porte. Produção, 18(1), 76-88.

(18) Ferreira, D.; Morabito, R. \& Rangel, S. (2008). Solution approaches for the soft drink integrated production lot sizing and scheduling problem. European Journal of Operational Research, doi: 10.1016/j.ejor.2008.03.035.

(19) FINEP (Financiadora de Estudos e Projetos) (2004). Relatório Setorial Final - Setor: Insumos Suínos. Autor: Giuliana Santini. Disponível em: <http://www.finep.gov.br/ PortalDPP/relatorio_setorial_final/relatorio_setorial_final_insumos_suinos $>$. Acessado em: 16/10/2006.

(20) Fleischmann, B. (1994). The discrete lot-sizing and scheduling problem with sequencedependent setup-costs. European Journal of Operational Research, 75, 395-404.

(21) Fleischmann, B. \& Meyr, H. (1997). The general lot sizing and scheduling problem. OR Spectrum, 19(1), 11-21.

(22) França, P.M.; Armentano, V.A.; Berretta, R.E. \& Clark, A.R. (1997). A heuristic method for lot-sizing in multi-stage systems. Computers \& Operations Research, 24(9), 861-874.

(23) Frieze, A.M. \& Dyer, M.E. (1990). On patching algorithms for random asymmetric travelling salesman problem. Mathematical Programming, 46, 361-378.

(24) Frieze, A.M.; Karp, R.M. \& Reed, B. (1995). When is the assignment bound tight for the asymmetric traveling-salesman problem? SIAM Journal on Computing, 24(3), 484-493.

(25) Gershwin, S. (1994). Manufacturing systems engineering. Prentice Hall, New Jersey.

(26) Glover, F.; Gutin, G.; Yeo, A. \& Zverovich, A. (2001). Construction heuristics for the asymmetric TSP. European Journal of Operational Research, 129, 555-568.

(27) Graham, E.L.; Lawler, E.L.; Lenstra, J.K. \& Rinnooy Kan, A.H.G. (1979). Optimization and approximation in deterministic sequencing and scheduling: a survey. Annals of Discrete Mathematics, 5, 287-326.

(28) Graves, S.C.; Rinnooy Kan, A.H.G. \& Zipkin, P.H. (1993). Logistics of Production and Inventory. Handbooks in Operations Research and Management Science, vol. 4, NorthHolland, Amsterdam.

(29) Gutin, G.; Yeo, A. \& Zverovitch, A. (2002). Exponential Neighborhoods and Domination Analysis for the TSP. In: The Traveling Salesman Problem and its Variations [edited by G. Gutin and A. Punnen], Kluwer, Dordrecht. 
(30) Haase, K. (1996). Capacitated Lot-Sizing with Sequence Dependent Setup Costs. OR Spectrum, 18, 51-59.

(31) Haase, K. \& Kimms, A. (2000). Lot Sizing and Scheduling with Sequence Dependent Setup Costs and Times and Efficient Rescheduling Opportunities. International Journal of Production Economics, 66, 159-169.

(32) Hax, A. \& Candea, D. (1984). Production and inventory management. Prentice-Hall, Englewood Cliffs, N.J.

(33) IFIF (International Feed Industry Federation) (2006). A Global Perspective on the Animal Feed Industry. Disponível em: <http://www.ifif.org/files/WorldFeedOverview.ppt>. Acessado em: 21/07/2006.

(34) Johnson, D.S.; Gutin, G.; McGeoch, L.A.; Yeo, A.; Zhang, W. \& Zverovich, A. (2002). Experimental analysis of heuristics for the asymmetric travelling salesman problem algorithms, instance generators and tests. In: The Traveling Salesman Problem and its Variations [edited by G. Gutin and A.P. Punnen], Kluwer.

(35) Jonhson, L.A. \& Montgomery, D.C. (1974). Operations research in production planning, scheduling and inventory control. Wiley, New York.

(36) Junger, M.; Reinelt, G. \& Rinaldi, G. (1995). The traveling salesman problem. In: Network Models [edited by M.O. Ball, T.L. Magnanti, C.L. Monma and G.L. Nemhauser], Handbooks in Operations Research and Management Science, vol. 7, North-Holland, chapter 4, 225-330.

(37) Karimi, B.; Ghomi, S.M.T.F. \& Wilson, J.M. (2003). The capacitated lot sizing problem: a review of models and algorithms. Omega International Journal of Management Science, 31(5), 365-378.

(38) Karp, R.M. (1979). A patching algorithm for the nonsymmetric traveling-salesman problem. SIAM Journal on Computing, 8(4), 561-573.

(39) Karp, R.M. \& Steele, J.M. (1985). Probabilistic analysis of heuristics. In: The Traveling Salesman Problem - A Guided Tour of Combinatorial Optimization [edited by E.L. Lawler, J.K. Lenstra, A.H.G. Rinnooy Kan and D.B. Shmoys], Wiley, Chichester.

(40) Laguna, M.A. (1999). Heuristic for Production Scheduling and Inventory Control in the presence of Sequence-Dependent Setup Times. Internal Research Report. University of Colorado.

(41) Laporte, G. (1992). The traveling salesman problem: An overview of exact and approximate algorithms. European Journal of Operational Research, 59, 231-247.

(42) Laporte, G. \& Nobert, Y. (1980). A Cutting Planes Algorithm for the m-Salesmen Problem. The Journal of the Operational Research Society, 31(11), 1017-1023.

(43) Lawler, E.L.; Lenstra, J.K.; Rinnooy Kan, A.H.G. \& Shmoys, D.B. (1985). The Traveling Salesman Problem - A Guided Tour of Combinatorial Optimization. Wiley, Chichester.

(44) Lawler, E.L.; Lenstra, J.K.; Rinnooy Kan, A.H.G. \& Shmoys, D.B. (1993). Sequencing and scheduling: Algorithms and complexity. In: Logistics of Production and Inventory [edited by S.C. Graves, A.H.G. Rinnooy Kan and P.H. Zipkin], Handbooks in Operations Research and Management Science, vol. 4, North-Holland, 445-522. 
(45) Luche, J.R. \& Morabito, R. (2005). Otimização na programação da produção de grãos eletrofundidos: Um estudo de caso. Gestão \& Produção, 12(1), 135-149.

(46) Maes, J. \& van Wassenhove, L.N. (1988). Multi-item single-level capacitated dynamic lot-sizing heuristics: a general review. Journal of the Operational Research Society, 39, 991-1004.

(47) Meyr, H. (2000). Simultaneous lot sizing and scheduling by combining local search with dual reoptimization. European Journal of Operational Research, 120, 311-326.

(48) Meyr, H. (2002). Simultaneous lot sizing and scheduling on parallel machines. European Journal of Operational Research, 139, 277-292.

(49) Nahmias, S. (1995). Production and Operations Analysis. Irwin, Homewood, IL.

(50) Orman, A.J. \& Williams, H.P. (2004). A survey of different integer programming formulations of the traveling salesman problem. Working paper LSEOR 04.67, London School of Economics, Dept of Operational Research, LSE, Houghton Street, London, WC2 2AE, UK.

(51) Padberg, M. \& Rinaldi, G. (1991). A Branch and Cut Algorithm for the Resolution of Large-scale Symmetric Traveling Salesman Problems. SIAM Review, 33(1), 66-100.

(52) Pataki, G. (2003). Teaching Integer Programming Formulations Using the Traveling Salesman Problem. SIAM review, 45, 116-123.

(53) Pekny, J.F. \& Miller, D.L. (1990). A parallel Branch and Bound Algorithm for Solving Large Asymetric Travelling Salesman Problems. Proceedings ACM, 56-62.

(54) Pinedo, M. (1995). Scheduling: theory, algorithms, and systems. Prentice-Hall, New Jersey.

(55) Pochet, Y. \& Wolsey, L.A. (2006). Production Planning by Mixed Integer Programming. Springer Series in Operations Research and Financial Engineering, New York.

(56) Potts, C. \& Kovalyov, M.Y. (2000). Scheduling with batching: a review. European Journal of Operational Research, 120(2), 228-249.

(57) Rangel, S. \& Ferreira, D. (2003). Um modelo de dimensionamento de lotes para uma fábrica de refrigerantes. Tema - Tendências em Matemática Aplicada e Computacional, $4(2), 237-246$

(58) Salomon, M.; Solomon, M.; Wassenhove, L. \& Dumas, Y. (1997). Solving the discrete lot sizing and scheduling problem with sequence dependent set-up costs and set-up times using the Travelling Salesman Problem with time windows. European Journal of Operational Research, 100, 494-513.

(59) SINDIRAÇÕES (Sindicato Nacional da Indústria de Alimentação Animal) (2006). Perfil 2005, Posicionamento da Indústria de Alimentação Animal. Disponível em: $<$ www.sindiracoes.org.br $>$. Acesso em: 21/07/2006.

(60) Toledo, C.F.; França, P.M.; Morabito, R. \& Kimms, A. (2007). Um modelo de otimização para o problema integrado de dimensionamento de lotes e programação da produção em fábricas de refrigerantes. Pesquisa Operacional, 27(1), 155-186.

(61) Toledo, C.F.; França, P.M.; Morabito, R. \& Kimms, A. (2008). Multi-population genetic algorithm to solve the synchronized and integrated two-level lot-sizing and scheduling problem. International Journal of Production Research, doi: $10.1080 / 00207540701675833$. 
(62) Toledo, F.M.B. \& Armentano, V.A. (2006). A Lagrangian-based heuristic for the capacitated lot-sizing problem in parallel machines. European Journal of Operational Research, 175(2), 1070-1083.

(63) Toso, E.A.V. (2008). Dimensionamento e Sequenciamento de Lotes de Produção na Indústria de Suplementos para Nutrição Animal. 191p. Tese de Doutorado (Departamento de Engenharia de Produção), Universidade Federal de São Carlos, São Carlos.

(64) Toso, E.A.V.; Morabito, R. \& Clark, A.R. (2007). Lot-Sizing and Sequencing Optimisation at an Animal-Feed Plant. Submetido para publicação.

(65) Toso, E.A.V. \& Morabito, R. (2005). Otimização no dimensionamento e sequenciamento de lotes de produção: estudo de caso numa fábrica de rações. Gestão \& Produção, 12(2), 203-217.

(66) Trigeiro, W.W.; Thomas, L.J. \& McClain, J.O. (1989). Capacitated lot sizing with setup times. Management Science, 35(3), 353-366.

(67) Williams, P. (1993). Model Building in Mathematical Programming. John Wiley \& Sons, NY.

(68) Winston, W. (1991). Operations Research: Applications and algorithms. PWS-Kent, Boston.

(69) Wolsey, L.A. (1998). Integer Programming. Wiley, New York.

(70) Zhang, W. (1993). Truncated Branch-and-Bound: A Case Study on the Asymmetric TSP. Spring Symposium on AI and NP-Hard Problems, 160-166.

(71) Zhang, W. (1997). A note on the complexity of the asymmetric travelling salesman problem. Operations Research Letters, 20, 31-38. 